ACCEPTED MANUSCRIPT

\title{
Far field diffraction of gratings with two roughness levels
}

To cite this article before publication: Francisco Jose Torcal-Milla et al $2019 \mathrm{~J}$. Opt. in press https://doi.org/10.1088/2040-8986/ab2bdf

\section{Manuscript version: Accepted Manuscript}

Accepted Manuscript is "the version of the article accepted for publication including all changes made as a result of the peer review process, and which may also include the addition to the article by IOP Publishing of a header, an article ID, a cover sheet and/or an 'Accepted Manuscript' watermark, but excluding any other editing, typesetting or other changes made by IOP Publishing and/or its licensors"

This Accepted Manuscript is @ 2019 IOP Publishing Ltd.

During the embargo period (the 12 month period from the publication of the Version of Record of this article), the Accepted Manuscript is fully protected by copyright and cannot be reused or reposted elsewhere.

As the Version of Record of this article is going to be / has been published on a subscription basis, this Accepted Manuscript is available for reuse under a CC BY-NC-ND 3.0 licence after the 12 month embargo period.

After the embargo period, everyone is permitted to use copy and redistribute this article for non-commercial purposes only, provided that they adhere to all the terms of the licence https://creativecommons.org/licences/by-nc-nd/3.0

Although reasonable endeavours have been taken to obtain all necessary permissions from third parties to include their copyrighted content within this article, their full citation and copyright line may not be present in this Accepted Manuscript version. Before using any content from this article, please refer to the Version of Record on IOPscience once published for full citation and copyright details, as permissions will likely be required. All third party content is fully copyright protected, unless specifically stated otherwise in the figure caption in the Version of Record.

View the article online for updates and enhancements. 


\title{
Far field diffraction of gratings with two roughness levels
}

\author{
F.J. Torcal-Milla ${ }^{1,3}$ and L.M. Sanchez-Brea ${ }^{2,3}$ \\ ${ }^{1}$ Departamento de Física Aplicada, Grupo de Tecnologías Ópticas \\ Láser, Instituto de Investigación en Ingeniería de Aragón (I3A), \\ Universidad de Zaragoza, 50018, Zaragoza (Spain) \\ ${ }^{2}$ Departamento de Óptica, Facultad de Física, Universidad \\ Complutense de Madrid, Plaza de las Ciencias 1, 28040, Madrid \\ (Spain) \\ ${ }^{3}$ Applied Optics Complutense Group, Universidad Complutense de \\ Madrid, Madrid (Spain) \\ E-mail: fjtorcal@unizar.es
}

\begin{abstract}
We investigate the far field diffraction process produced by diffraction gratings with two roughness levels such as steel tape gratings, which are used in environments where diffraction gratings made of glass are not possible. We obtain the analytical formulation describing the formation of diffraction orders in terms of the roughness parameters. Since roughness is stochastic, we use the mutual coherence function to propagate the field to the Fraunhofer regime. We conclude that diffraction orders exists when, at least, one correlation length of both roughness is larger than the period of the grating and the standard deviation on heights is around or larger than the illumination wavelength. Finally, we corroborate the analytical results with numerical simulations based of the Fast Fourier Transform, showing a high agreement.
\end{abstract}

Keywords: Diffraction, Diffraction gratings, Roughness, Far-field

PACS numbers: 42.25.Fx, 42.79.Dj,

AMS classification scheme numbers:

Submitted to: J. Opt. 


\section{Introduction}

A diffraction grating is an optical element that modulates periodically one or several properties of the incident beam. They are one of the most used optical components, as they are used in metrology, spectrometers, telescopes, and so on, [1]. Diffraction gratings have been analyzed from several points of view, [2]. Amplitude and phase gratings, which modulate the amplitude or the phase of the incident light respectively, are the most common diffraction gratings, but other types of gratings have been reported in the recent years, such as polarization gratings [3-5] or gratings that modulate the spatial-coherence state of light. About spatial-coherence state, it can be modulated by means of the stochastic rough surface on the grating. This fact has been reported for some approximations in [6-10]. Particularly, in $[8,11]$ the near field diffraction of rough gratings with one or two roughness levels is reported. Analytical equations showing the dependence of the self-imaging phenomenon on the parameters of the roughness are obtained. It reveals that self-images disappear for a certain distance forward due to the roughness when the grating has only one roughness level [8]. On the contrary, when the grating has two roughness levels, the self-imaging phenomenon does not appear just close to the grating but from a certain distance from it [11]. Self-images of the grating are replicas of the grating pattern that appears at multiples of the so-called Talbot distance $z_{T}=2 p^{2} / \lambda$, where $p$ is the period of the grating and $\lambda$ is the illumination wavelength, [12]. Now, if we pay attention to the far-field behavior of rough gratings, those with one roughness level have been reported in $[9,13,14]$. Particularly, in [9] diffraction orders are shown to appear as we increase the amount of roughness. In addition, a halo of scattered light appears around the zero-th order. In this work, we complete the analysis performed in [11] to the far-field approach, using the mutual coherence function to describe the reflectance of the grating and the Fraunhofer kernel to propagate the field to the far-field. The approach carried out along this manuscript is valid as long as the correlation length of roughness and the period of the grating were longer than the illumination wavelength. As it was pointed out, steel tape gratings manufactured by laser ablation on a steel substrate can be assumed as a particular case of the grating proposed in this manuscript [15, 16]. We conclude that diffraction orders appear when correlation length of at least one roughness level is larger than the period of the grating and the standard deviation on heights is around or larger than the illumination wavelength. Finally we corroborate the analytical formalism with numerical simulations. Both approaches result very similar, validating the obtained formulation.

\section{Theoretical approach}

Firstly, let us define a diffraction grating of period $p$ formed by strips with two roughness levels. One example could be a grating engraved by laser ablation on a steel substrate, commonly named steel tape grating. Due to manufacturing, a long range roughness is naturally present along two perpendicular directions on the steel tape before engraving and a short range roughness is intentionally produced by laser ablation to generate the strips that will form the diffraction grating. A confocal image of a steel tape grating can be observed in Figure 1. Both levels of roughness are defined by means of the correlation length and the standard deviation of heights. The long range roughness is characterized by two perpendicular correlation lengths, $T_{l, x}$ and $T_{l, y}$, and the standard deviation $\sigma_{l}$. On the other hand, we suppose that short range roughness is equal in both directions and then it is characterized by only one correlation length, $T_{s}$, and the standard deviation $\sigma_{s}$. Since the periodicity of the grating is along the $\mathrm{x}$-axis, we can consider the problem as one-dimensional, neglecting the effects produced by the roughness along y-axis on the diffraction pattern. 
Far field diffraction of gratings with two roughness levels

(a)

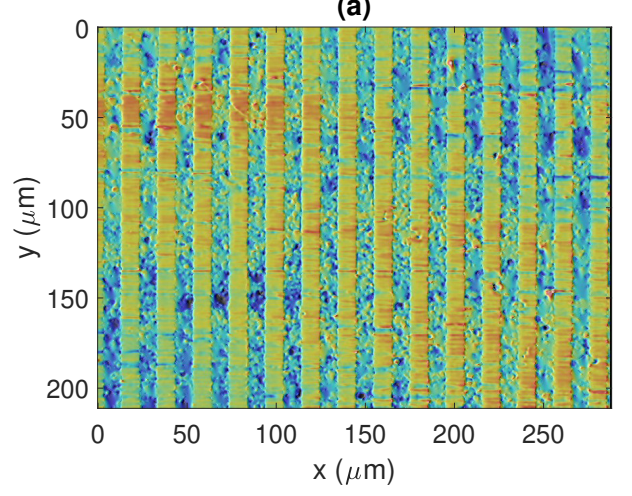

(b)

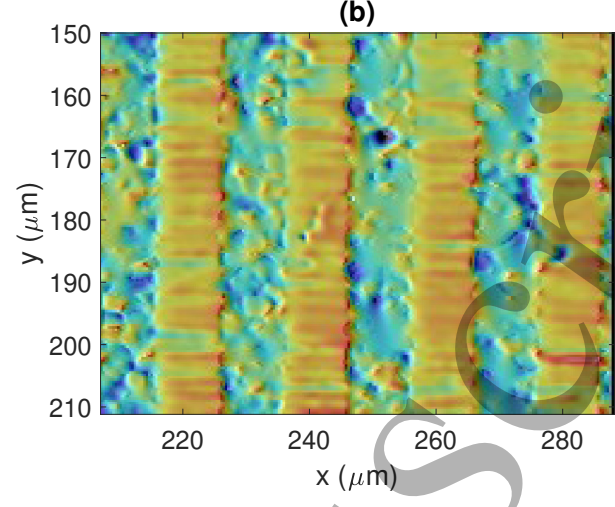

Figure 1. (a) 2D confocal image of a steel tape grating showing both roughness levels, (b) zoom of Figure1(a) to observe the short range roughness. The parameters of the grating are: $p=20 \mu \mathrm{m}, T_{l, x}=12.72 \mu \mathrm{m}, T_{l, y}=1.34 \mu \mathrm{m}, T_{s, x}=T_{s, y}=T_{s}=3.78 \mu \mathrm{m}, \sigma_{l}=0.13 \mu \mathrm{m}$, and $\sigma_{s}=0.23 \mu \mathrm{m}$. These parameters have been obtained from confocal microscope images.

Mathematically, the reflectance of the grating can be defined as the summation of two complementary gratings with rough strips

$$
r(x)=G(x) r_{l}(x)+G(x+p / 2) r_{s}(x),
$$

where $G(x)=\sum_{n} a_{n} \exp ($ inq $x)$ is the reflectance of a common amplitude Ronchi grating with $i$ the imaginary unit, $n$ entire numbers, $q=2 \pi / p, a_{n}=\kappa \operatorname{sinc}(n \kappa), \kappa=\alpha / p$, and $\alpha$ the fill-factor of the grating. It only depends on $x$ because the grating without roughness is defined unidirectional. $r_{l}(x)$ and $r_{s}(x)$ define the reflectance of the so-called long and short range roughness respectively. These two reflectances depend on the statistical parameters of the rough surfaces as $[17,18]$

$$
\begin{aligned}
& r_{l}(x)=\exp \left[-2 i k \zeta_{l}(x)\right] \\
& r_{s}(x)=\exp \left[-2 i k \zeta_{s}(x)\right],
\end{aligned}
$$

being $k=2 \pi / \lambda, \lambda$ the illumination wavelength and $\zeta(x)$ the topographies of both roughness, long and short. In order to perform a statistical analysis, let us consider the grating placed on a mobile system. For example, it can be part of an optical encoder or other metrological device. Without loss of generality, we assume normal distribution with mean value zero for both roughness, $w\left[\zeta_{j}(x)\right]=\exp \left[-\zeta_{j}^{2}(x) / 2 \sigma_{j}^{2}\right] / \sqrt{2 \pi} \sigma_{j}$ where $j=l, s$. The average reflectance can be expressed as $[17,18]$

$$
<r_{j}>=\int w\left(\zeta_{j}\right) \exp \left(-2 i k \zeta_{j}\right) d \zeta_{j}=\exp \left(-g_{j, x} / 2\right), \quad j=l, s,
$$

where $g_{j}=\left(2 k \sigma_{j}\right)^{2}$ and $<>$ denotes average. We have considered the standard deviation in heights equal in both directions for both roughness and then

$$
<r_{j}>=\exp \left[-\left(2 k \sigma_{j}\right)^{2} / 2\right], \quad j=l, s .
$$

Besides, we need the statistical relationship between two different points of the surface. Considering again Gaussian distribution with mean value zero and uncorrelation between both perpendicular directions, the $2 \mathrm{D}$ characteristic function can be expressed as $[17,18]$

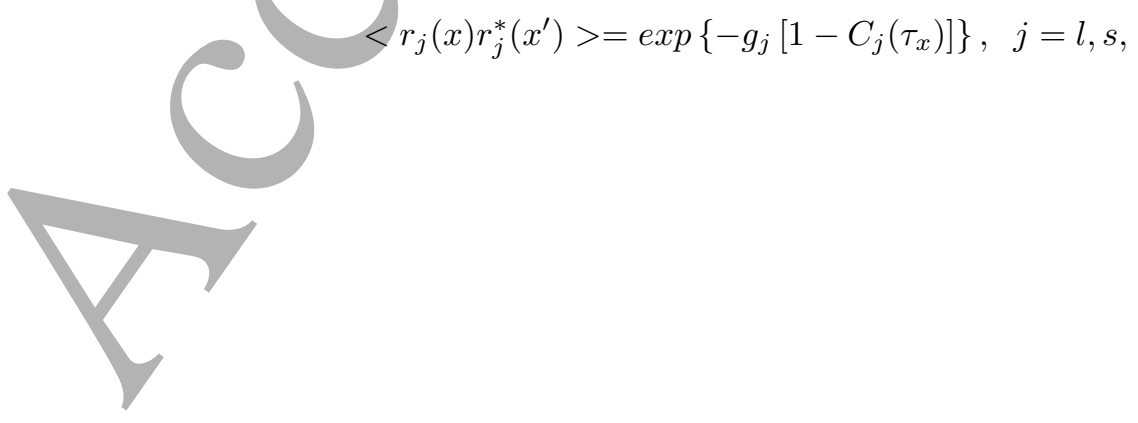


where the normalized correlation function is also assumed Gaussian $C_{j}\left(\tau_{x}\right)=\exp \left(-\tau_{j, x}^{2} / T_{j, x}^{2}\right)$, $\tau_{x}=x-x^{\prime}, T_{j, x}$ the correlation length, and $j=l, s$. Finally, the characteristic function of each roughness can be rewritten as

$$
<r_{j}(x) r_{j}^{*}\left(x^{\prime}\right)>=\exp \left(-g_{j}\right) \sum_{m_{j}=0}^{\infty} \frac{g_{j}^{m_{j}}}{m_{j} !} \exp \left[-m_{j} \frac{\tau_{x}^{2}}{T_{j, x}^{2}}\right], j=l, s .
$$

Considering that both roughness are statistically independent, then

$$
<r_{l}(x) r_{s}^{*}\left(x^{\prime}\right)>=<r_{l}^{*}\left(x^{\prime}\right) r_{s}(x)>=\exp \left[-\left(2 k \sigma_{l}\right)^{2} / 2\right] \exp \left[-\left(2 k \sigma_{s}\right)^{2} / 2\right] .
$$

Summarizing, the average reflectances and characteristic functions for both roughness are given by

$$
\begin{aligned}
& <r_{l}>=\exp \left[-\left(2 k \sigma_{l}\right)^{2} / 2\right] \\
& <r_{s}>=\exp \left[-\left(2 k \sigma_{s}\right)^{2} / 2\right] \\
& <r_{l} r_{l}^{*}>=\exp \left(-g_{l}\right) \sum_{m_{l}=0}^{\infty} \frac{g_{l}^{m_{l}}}{m_{l} !} \exp \left(-m_{l} \frac{\tau_{x}^{2}}{T_{l, x}^{2}}\right) \\
& <r_{s} r_{s}^{*}>=\exp \left(-g_{s}\right) \sum_{m_{s}=0}^{\infty} \frac{g_{s}^{m_{s}}}{m_{s} !} \exp \left(-m_{s} \frac{\tau_{x}^{2}}{T_{s}^{2}}\right) .
\end{aligned}
$$

Following, we consider Gaussian illumination, $U_{0}(x)=A_{0} \exp \left(-x^{2} / \omega^{2}\right)$, where $\omega$ is the width. The field after reflecting from the grating is $U_{1}(x)=U_{0}(x) r(x)$. The grating has stochastic behavior and then we use the mutual coherence function to describe the scattering process and propagation of light after impinging the grating. It is defined/as $J_{1}\left(x, x^{\prime}\right)=<U_{1}(x) U_{1}^{*}\left(x^{\prime}\right)>$. Since the only stochastic process is produced by the rough topography of the grating, from Equation 1, the normalized mutual coherence function at the grating plane results in

$$
\begin{aligned}
\overline{J_{1}\left(x, x^{\prime}\right)} & =U_{0}\left(x, x^{\prime}\right)\left[G(x) G^{*}\left(x^{\prime}\right)<r_{l}(x) r_{l}^{*}\left(x^{\prime}\right)>\right. \\
& +G(x) G^{*}\left(x^{\prime}+p / 2\right)<r_{l}(x) r_{s}^{*}\left(x^{\prime}\right)> \\
& +G(x+p / 2) G^{*}\left(x^{\prime}\right)<r_{l}^{*}\left(x^{\prime}\right) r_{s}(x)> \\
& \left.+G(x+p / 2) G^{*}\left(x^{\prime}+p / 2\right)<r_{s}(x) r_{s}^{*}\left(x^{\prime}\right)>\right],
\end{aligned}
$$

where $\overline{J_{1}\left(x, x^{\prime}\right)}=J_{1}\left(x, x^{\prime}\right) / A_{0}^{2}$.

Substituting Equations 7 and 8 into Equation 9, the initial mutual coherence function is rewritten as

$$
\begin{aligned}
\overline{J_{1}\left(x, x^{\prime}\right)} & =U_{0}\left(x, x^{\prime}\right)\left[G(x) G^{*}\left(x^{\prime}\right) e^{-g_{l}} \sum_{m_{l}=0}^{\infty} \frac{g_{l}^{m_{l}}}{m_{l} !} e^{-m_{l}\left(\frac{\tau_{x}}{T_{l, x}}\right)^{2}}\right. \\
& +\left[G(x) G^{*}\left(x^{\prime}+p / 2\right)+G(x+p / 2) G^{*}\left(x^{\prime}\right)\right] e^{-\frac{\left(2 k \sigma_{l}\right)^{2}}{2}} e^{-\frac{\left(2 k \sigma_{s}\right)^{2}}{2}} \\
& \left.+G(x+p / 2) G^{*}\left(x^{\prime}+p / 2\right) e^{-g_{s}} \sum_{m_{s}=0}^{\infty} \frac{g_{s}^{m_{s}}}{m_{s} !} e^{-m_{s} \frac{\tau_{x}^{2}}{T_{s}^{2}}}\right] .
\end{aligned}
$$

Considering that $G(x+p / 2)=\sum_{n} a_{n} \exp ($ inqx $) \exp ($ in $\pi)$ and $G^{*}\left(x^{\prime}+p / 2\right)=$ $\sum_{n^{\prime}} a_{n^{\prime}}^{*} \exp \left(-i n^{\prime} q x^{\prime}\right) \exp \left(-i n^{\prime} \pi\right)$, we may rewrite the normalized mutual coherence function at 
Far field diffraction of gratings with two roughness levels

(a)

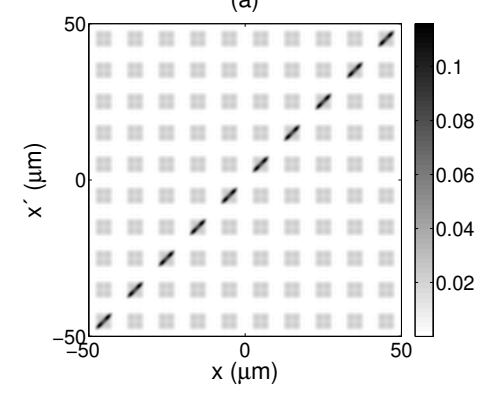

(b)

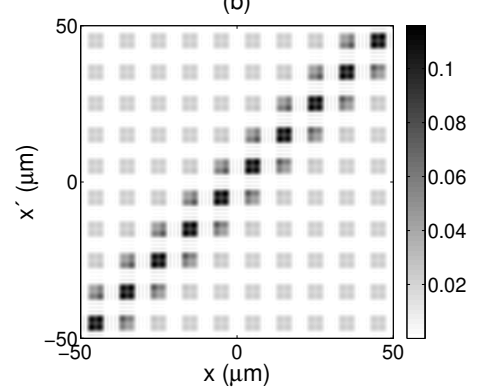

(c)

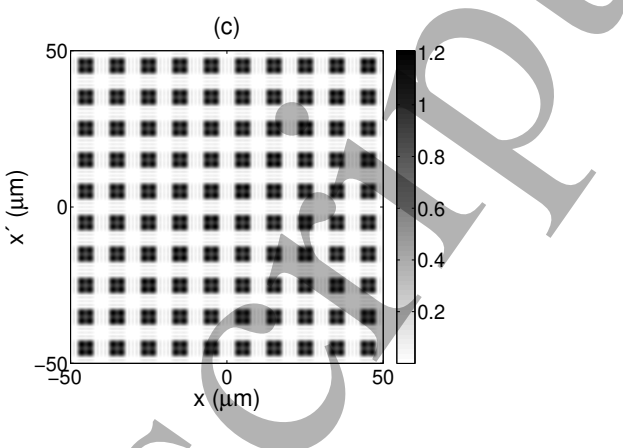

Figure 2. Examples of analytical normalized mutual coherence function at the grating plane calculated by using Equation 11. The used parameters are: $p=10 \mu \mathrm{m}, \lambda=632.8 \mathrm{~nm}, m=0,1$, $n=-3,-1,0,1,3$. (a) $\sigma_{s}=0.1 \mu \mathrm{m}, T_{s}=1 \mu \mathrm{m}, \sigma_{l}=1 \mu \mathrm{m}, T_{l}=100 \mu \mathrm{m}$, (b) $\sigma_{s}=0.1 \mu \mathrm{m}$, $T_{s}=10 \mu \mathrm{m}, \sigma_{l}=1 \mu \mathrm{m}, T_{l}=100 \mu \mathrm{m}$, and (c) $\sigma_{s}=0.01 \mu \mathrm{m}, T_{s}=10 \mu \mathrm{m}, \sigma_{l}=1 \mu \mathrm{m}, T_{l}=100 \mu \mathrm{m}$.

the grating plane as

$$
\begin{aligned}
\overline{J_{1}\left(x, x^{\prime}\right)} & =U_{0}\left(x, x^{\prime}\right)\left[\sum_{n, n^{\prime}} a_{n} a_{n^{\prime}}^{*} \exp \left[i q\left(n x-n^{\prime} x^{\prime}\right)\right] e^{-g_{l}} \sum_{m_{l}=0}^{\infty} \frac{g_{l}^{m_{l}}}{m_{l} !} e^{-m_{l} \frac{\tau_{x}^{2}}{T_{l, x}^{2}}}\right. \\
& +\sum_{n, n^{\prime}} a_{n} a_{n^{\prime}}^{*} \exp \left[i q\left(n x-n^{\prime} x^{\prime}\right)\right]\left[\exp \left(-i n^{\prime} \pi\right)+\exp (i n \pi)\right] e^{-\frac{g_{l}}{2}} e^{-\frac{g_{s}}{2}} \\
& \left.+\sum_{n, n^{\prime}} a_{n} a_{n^{\prime}}^{*} \exp \left[i q\left(n x-n^{\prime} x^{\prime}\right)\right] \exp \left[i \pi\left(n-n^{\prime}\right)\right] e^{-g_{s}} \sum_{m_{s}=0}^{\infty} \frac{g_{s}^{m_{s}}}{m_{s} !} e^{-m_{s} \frac{\tau_{x}^{2}}{T_{s}^{2}}}\right](11)
\end{aligned}
$$

First and last terms are the same but considering different roughness and an exponential correcting factor that corresponds to the lateral shifting between both considered gratings. We show in Figure 2 three examples of the initial normalized mutual coherence function calculated by using Equation 11. The behavior of this function depends on the difference between roughness parameters of both roughness levels. In Figure 2(a) standard deviations are relatively close and correlation lengths are relatively remote. Then the normalized mutual coherence function only shows correlation with itlself. On the other hand, in Figure 2(b) standard deviations and correlation lengths are relatively close and the normalized mutual coherence function shows correlation also by displacing it around itself a little bit. Finally, in Figure 2(c) standard deviations are relatively remote and correlation lengths are relative close. In this case the mutual intensity function reaches indistinguishable maxima by displacing the grating a entire number of periods. As we will explain following, the diagonal of each figure would correspond to the intensity at the grating plane.

Following, the far field mutual intensity function can be obtained as

$$
\frac{}{J_{2}\left(x_{2}, x_{2}^{\prime}\right)} \propto \int_{-\infty}^{+\infty} \int_{-\infty}^{+\infty} \overline{J_{1}\left(x, x^{\prime}\right)} e^{-i \frac{k}{z} x_{2}^{\prime} x^{\prime}} e^{+i \frac{k}{z} x_{2} x} d x d x^{\prime}
$$

resulting in

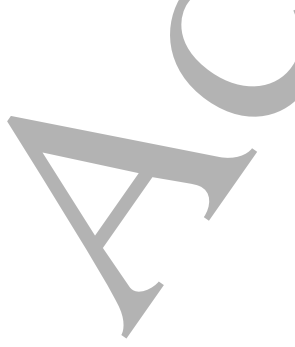

$$
\overline{J_{2}\left(x_{2}, x_{2}^{\prime}\right)} \propto \sum_{m_{l}=1}^{M_{l}} \frac{g_{l}^{m_{l}} e^{-g_{l}} T_{l}}{\sqrt{2 m_{l}} m_{l} !} \sum_{n, n^{\prime}=-N}^{N} a_{n} a_{n^{\prime}} \exp \left[-\frac{\left(n-n^{\prime}\right)^{2} q^{2}}{4\left(T_{l}^{2} / m_{l} \omega^{4}\right)}\right]
$$




$$
\begin{aligned}
& \times \exp \left[-\frac{\left(k x_{2}-n q z\right)^{2}}{4 z^{2}\left(2 m_{l} / T_{l}^{2}\right)}\right] \exp \left[-\frac{\left(k x_{2}^{\prime}-n^{\prime} q z\right)^{2}}{4 z^{2}\left(2 m_{l} / T_{l}^{2}\right)}\right] \\
& +\sum_{m_{s}=1}^{M_{s}} \frac{g_{s}^{m_{s}} e^{-g_{s}} T_{s}}{\sqrt{2 m_{s}} m_{s} !} \sum_{n, n^{\prime}=-N}^{N} a_{n} a_{n^{\prime}} \exp \left[-\frac{\left(n-n^{\prime}\right)^{2} q^{2}}{4\left(T_{s}^{2} / m_{s} \omega^{4}\right)}\right] \\
& \times \exp \left[-\frac{\left(k x_{2}-n q z\right)^{2}}{4 z^{2}\left(2 m_{s} / T_{s}^{2}\right)}\right] \exp \left[-\frac{\left(k x_{2}^{\prime}-n^{\prime} q z\right)^{2}}{4 z^{2}\left(2 m_{s} / T_{s}^{2}\right)}\right]
\end{aligned}
$$

where $T_{l}=T_{l, x}$ for simplicity. Notice that the summations on $m_{l}$ and $m_{s}$ starts from 1 instead from 0 and we have truncated the summations over $m_{l}$ and $m_{s}$ at $M_{l}$ and $M_{s}$ respectively. The intensity at the far field is obtained just as $\overline{I_{2}\left(x_{2}\right)}=\overline{J_{2}\left(x_{2}, x_{2}\right)}$. Besides, we may extend the illumination to plane wave illumination by performing $\omega \rightarrow \infty$. Thus, the normalized mean intensity is given by

$$
\begin{aligned}
\overline{I_{2}\left(x_{2}\right)} & \propto \sum_{m_{l}=1}^{M_{l}} \frac{g_{l}^{m_{l}} e^{-g_{l}} T_{l}}{\sqrt{2 m_{l}} m_{l} !} \sum_{n=-N}^{N} a_{n}^{2} \exp \left[-\frac{T_{l}^{2}(k \theta-n q)^{2}}{4 m_{l}}\right] \\
& +\sum_{m_{s}=1}^{M_{s}} \frac{g_{s}^{m_{s}} e^{-g_{s}} T_{s}}{\sqrt{2 m_{s}} m_{s} !} \sum_{n=-N}^{N} a_{n}^{2} \exp \left[-\frac{T_{s}^{2}(k \theta-n q)^{2}}{4 m_{s}}\right],
\end{aligned}
$$

where we have also defined the angular coordinates, $\theta=x_{2} / z$ and we have applied $n^{\prime}=n$ since diffraction orders do not overlap usually/in the far field.) As can be observed, the total average intensity is given by the summation of two terms which correspond to the superposition of intensities produced by two independent rough gratings with long and short range roughness respectively. The width of the diffraction orders is mainly controlled by the correlation lengths of the roughness, $T_{l}$ and $T_{s}$, and the power of all diffraction orders is weighted by the same multiplying factor. On the other hand, terms with $m_{l}>1$ and $m_{s}>1$ are neglictible and Equation 14 can be simplified resulting

$$
\begin{aligned}
\overline{I_{2}\left(x_{2}\right)} & \propto \frac{g_{l} e^{-g_{l}} T_{l}}{\sqrt{2}} \sum_{n=-N}^{N} a_{n}^{2} \exp \left[-\frac{T_{l}^{2}(k \theta-n q)^{2}}{4}\right] \\
& +\frac{g_{s} e^{-g_{s}} T_{s}}{\sqrt{2}} \sum_{n=-N}^{N} a_{n}^{2} \exp \left[-\frac{T_{s}^{2}(k \theta-n q)^{2}}{4}\right] .
\end{aligned}
$$

Figure 3 shows two examples of intensity for different values of both roughness parameters. We also give the diffraction efficiency of the first diffraction order, $\eta_{ \pm 1}$.

In the limit case, when we consider the same statistical parameters for both gratings, the intensity does not result a constant but

$$
\overline{I_{2}\left(x_{2} ; l=s\right)} \propto \sqrt{2} g e^{-g} T \sum_{n=-N}^{N} a_{n}^{2} \exp \left[-\frac{(k \theta-n q)^{2}}{4 / T^{2}}\right] .
$$

The correlation length, $T$, and standard deviation on heights, $\sigma$, of roughness affect equally to all diffraction orders. Both parameters affect to the power of the diffraction orders but only the correlation length affect to their width. In a first sight, we could think that since all the strips have the same parameters, the grating disappear and only a scattering effect is produced. When 
(a)

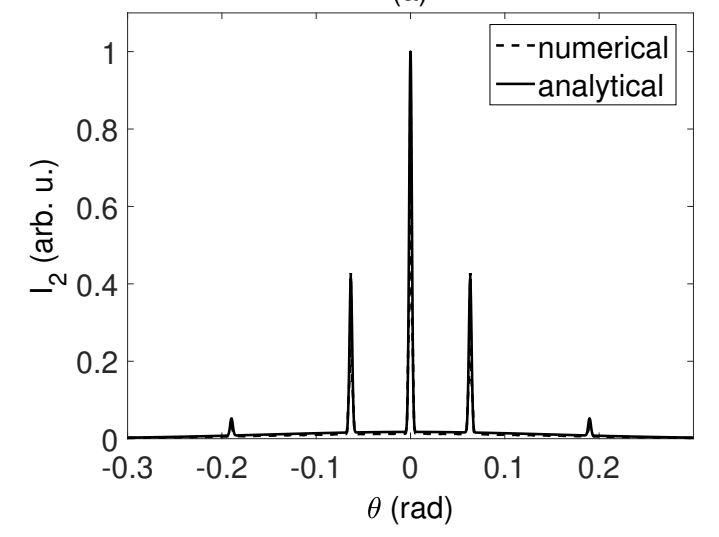

(b)

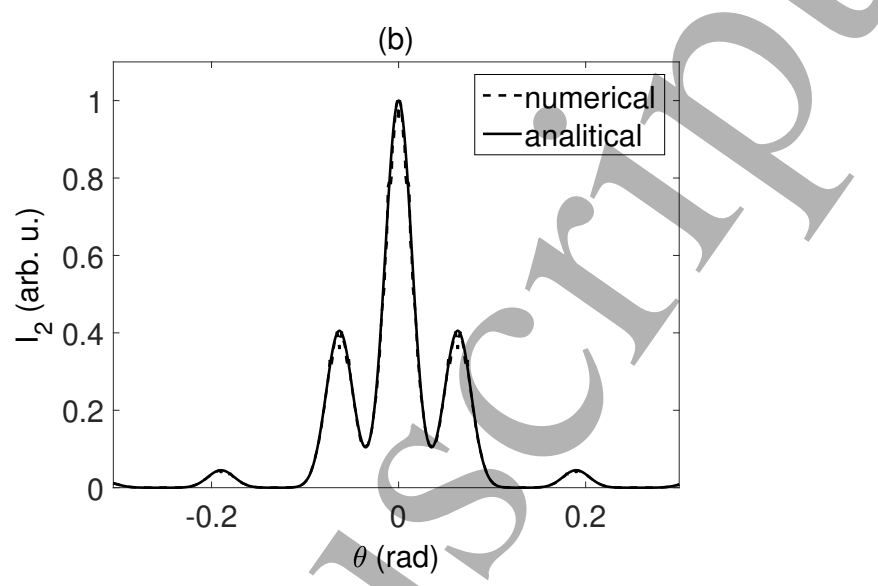

Figure 3. Analytical (calculated using Equation 14) and numerical intensity at the far field. The used parameters are: $p=10 \mu \mathrm{m}, \lambda=632.8 \mathrm{~nm}, M_{l}=M_{s}=10$. (a) $\sigma_{s}=1 \mu \mathrm{m}, T_{s}=1 \mu \mathrm{m}$, $\sigma_{l}=1 \mu \mathrm{m}, T_{l}=100 \mu \mathrm{m}, \eta_{ \pm 1}=0.42$, (b) $\sigma_{s}=1 \mu \mathrm{m}, T_{s}=100 \mu \mathrm{m}, \sigma_{l}=0.1 \mu \mathrm{m}, T_{l}=100 \mu \mathrm{m}$, $\eta_{ \pm 1}=0.40$. Numerical results represent the mean value over 50 realizations.

the correlation length is longer than the grating period, although the statistical parameters are the same, the grating structure is present, as can be seen in Figure 4(a)-(c). On the other hand, when the correlation length of roughness is shorter than the period of the grating, the exponential terms in Equation 16 are very wide and overlap, Figure 4(d)-(f), corresponding to the scattering of a rough surface (without grating).

\section{Numerical simulations}

In this section we perform several numerical simulations to corroborate the analytical formalism obtained in the previous section. First of all, we generate the proposed diffraction grating in a similar fashion as it is performed in [11], where two complementary gratings with different roughness are matematically added. The grating is formed by interleaved slits with surficial roughness as it is shown in Figure 1 and Figures 4(a) and 4(d). We have used the Fast Fourier Transform to determine the intensity at the far field. Besides, since the analytical formalism has been obtained over an ensemble of realizations, we need to perform also a numerical average. Thus, we perform the averaging process over an ensemble of 50 gratings. We show in Figure 3 both analytical and numerical mean intensity showing a great agreement between them. In Figure 3(a) we observe a kind of scattering centered at zero angle which does not affect to the width of the diffraction orders. On the other side, in Figure 3(b) the width of the orders increases making them to partially overlap in some cases.

As a particular case, we show in Figure 4 two diffraction gratings with the same statistical parameters for both roughness. In the first case, Figure 4(a), the correlation length is around the value of the period of the grating or longer. In this case, we obtain wide diffraction orders performing an average over several realizations. In the second case, Figure 4(d), the correlation length of the-roughness is shorter than the period of the grating. If we look at the zoom shown into the figure, it is possible to see a certain periodical structure that remains correlation between odd fringes and even fringes separately. In this case we do not obtain diffraction orders but the 
diffraction pattern typical of a rough surface without grating. As we can see in Figure 4(c) and (f), the numerical results also corroborate in these cases the obtained analytical formalism given by Equation 16.

\section{Conclusions}

In this work, we obtain analytical description of the scattering process produced by diffraction gratings of two roughness levels at the far field. We show how diffraction process occurs due to roughness and how the amount of roughness affects to the diffraction orders formation. Since roughness is random, we use the mutual coherence function to propagate the field from the grating plane to the far field. This type of grating can explain the behaviour of steel tape gratings which has two kinds of roughness, one of them due to the proper surface of the steel and the other one due to the engraving process. Steel tape gratings are commonly used in environments where glass diffraction gratings are not possible. Finally, we corroborate the theoretical results with numerical simulations based on the Fast Fourier Transform algorithm, showing a very good agreement. If both surface roughness have correlation lengths shorter than the period of the grating, the far field diffraction pattern corresponds to a typical scattering pattern of a rough surface since diffraction orders totally overlap at the far field. On the other hand, when at least one surface roughness has correlation length around or longer than the period of the grating, diffraction orders appear but they are wider than for a common binary diffraction grating.

\section{Acknowledgments}

This work has been performed with the funding of Gobierno de Aragon-Fondo Social Europeo (Grupo de Tecnología Óptica Láser - E44_17R) and Ministerio de Economía y Competitividad (MINECO) "Ecograb" of "Programa Estatal de Investigacion, Desarrollo e Innovacion Orientada a los Retos de la Sociedad" (RTC-2016-5277-5); co-financed with structural funds of the European Union.

\section{References}

[1] E. Popov and E. G. Loewen, Diffraction gratings and applications, Marcel Dekker, New York, 1997.

[2] C. Palmer, Diffraction Grating Handbook 5th edn (Rochester, NY: Richardson Grating Laboratory), 2000.

[3] F. Gori, "Measuring stokes parameters by means of a polarization grating," Optics letters 24(9), pp. 584-586, 1999.

[4] C. G. Someda, "Far field of polarization gratings," Optics letters 24(23), pp. 1657-1659, 1999.

[5] C. Oh and M. J. Escuti, "Achromatic diffraction from polarization gratings with high efficiency," Optics letters 33(20), pp. 2287-2289, 2008.

[6] F. J. Torcal-Milla, L. M. Sanchez-Brea, and E. Bernabeu, "Talbot effect with rough reflection gratings," Applied optics 46(18), pp. 3668-3673, 2007.

[7] F. J. Torcal-Milla and L. M. Sanchez-Brea, "Gaussian-schell-model beams propagating through rough gratings," JOSA A 28(3), pp. 308-313, 2011. 
(a)

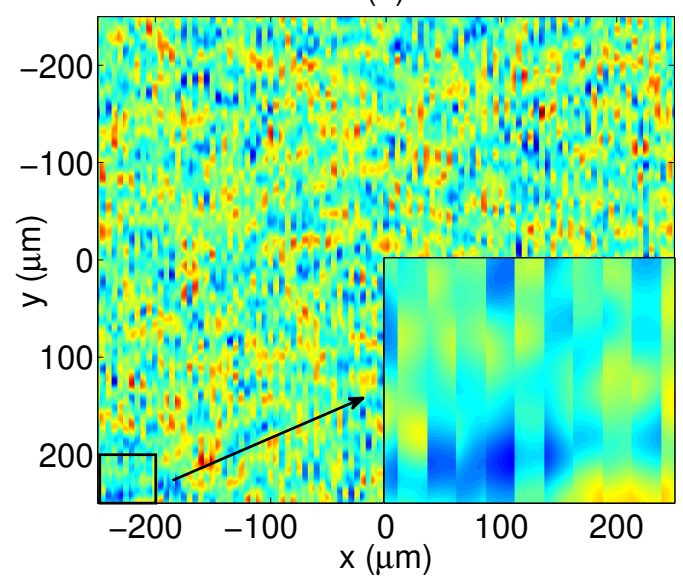

(b)

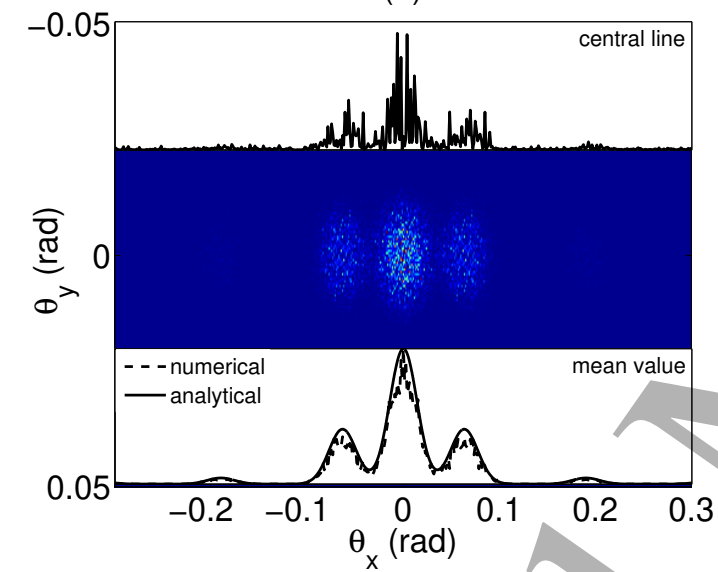

(c)

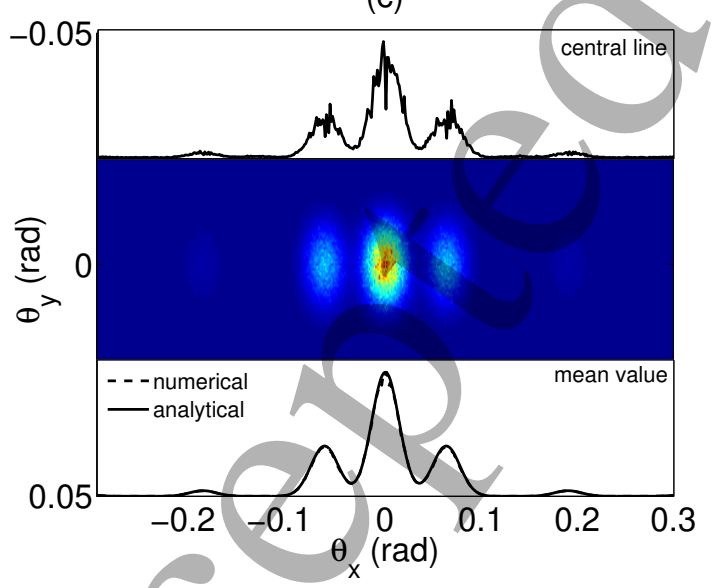

(d)

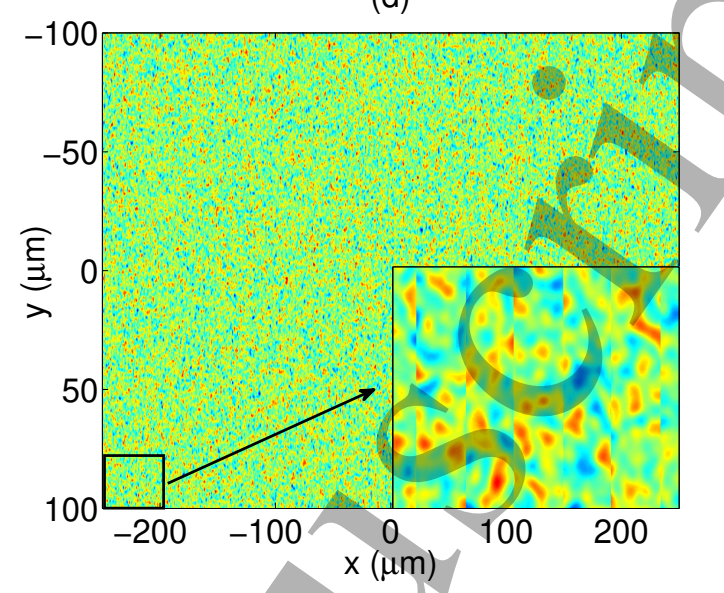

(e)

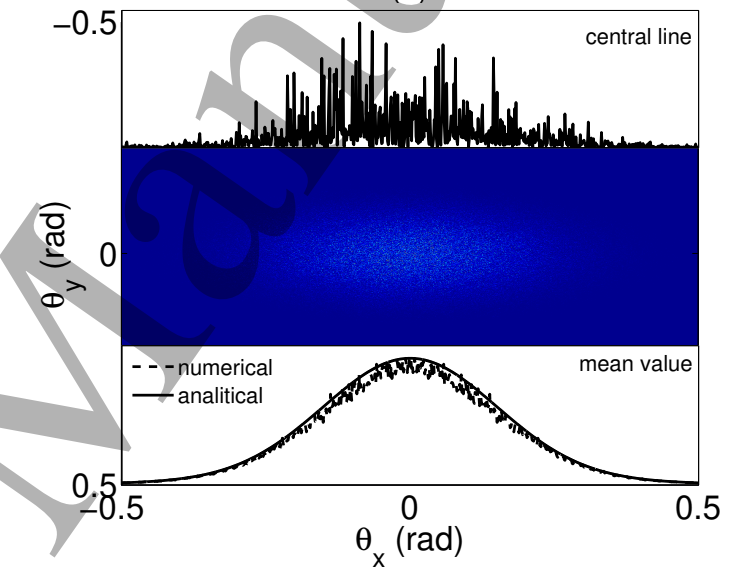

(f)

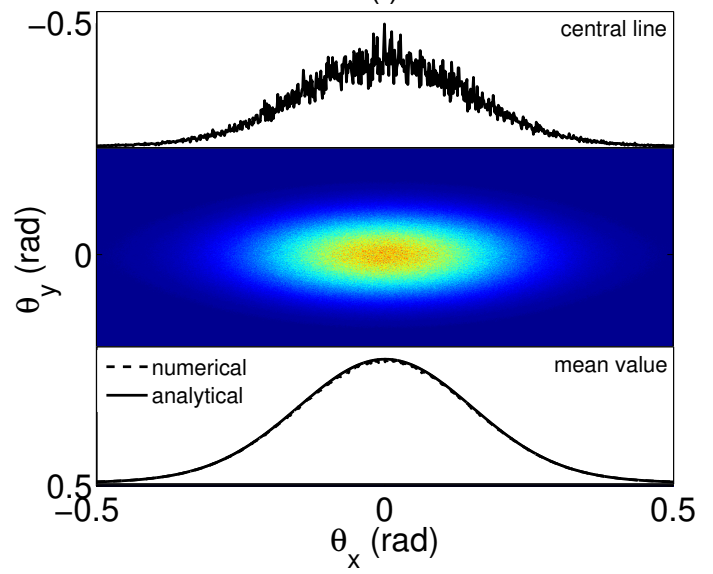

Figure 4. (a) Example of rough grating with the same parameters for both roughness $\left(\sigma_{s}=\sigma_{l}=1 \mu \mathrm{m}, T_{s}=T_{l}=10 \mu \mathrm{m}\right)$, (b) far field intensity for one realization of the grating (a), (c) average far field intensity over an ensemble of 50 realizations of the grating (a), (d) Example of rough grating with the same parameters for both roughness $\left(\sigma_{s}=\sigma_{l}=1 \mu \mathrm{m}\right.$, $T_{s}=T_{l}=1 \mu \mathrm{m}$ ), (e) far field intensity for one realization of the grating (d), (f) average far field intensity over an ensemble of 50 realizations of the grating $(\mathrm{d})$. The period of the grating is $p=10 \mu \mathrm{m}$, the illumination wavelength is $\lambda=632.8 \mathrm{~nm}$ and the summations for the roughness have been taken only up to the first term, $M_{l}=M_{s}=1$, since it is the most powerful. The analytical results have been calculated by using Equation 16. The numerical results have been obtained by generating the gratings with the desired roughness parameters and using the Fast Fourier Transform algorithm. 
[8] F. J. Torcal-Milla, L. M. Sanchez-Brea, and E. Bernabeu, "Self-imaging of gratings with rough strips," JOSA A 25(10), pp. 2390-2394, 2008.

[9] L. M. Sanchez-Brea, F. J. Torcal-Milla, and E. Bernabeu, "Far field of gratings with rough strips," JOSA A 25(4), pp. 828-833, 2008.

[10] L. M. Sanchez-Brea, F. J. Torcal-Milla, and E. Bernabeu, "Talbot effect in metallic gratings under gaussian illumination," Optics communications 278(1), pp. 23-27, 2007.

[11] L. M. Sanchez-Brea and F. J. Torcal-Milla, "Self-imaging of gratings with two roughness levels," Optics Communications 285(1), pp. 13-17, 2012.

[12] K. Patorski, "I the self-imaging phenomenon and its applications," in Progress in optics, 27, pp. 1-108, Elsevier, 1989.

[13] P. Tran and V. Celli, "Monte carlo calculation of backscattering enhancement for a randomly rough grating," JOSA A 5(10), pp. 1635-1637, 1988.

[14] T. Michel, "Resonant light scattering from weakly rough random surfaces and imperfect gratings," JOSA A 11(6), pp. 1874-1885, 1994.

[15] F. J. Salgado-Remacha, F. J. Torcal-Milla, L. M. Sanchez-Brea, and E. Bernabeu, "Use of steel substrates in diffractive optics: near field of high surface quality steel tape gratings," Optics and Lasers in Engineering 49(3), pp. 356-360, 2011.

[16] N. Pan, W. Ma, and J. Huang, "The error analysis and correcting of scale tape grating encoder," in International Symposium on Photoelectronic Detection and Imaging 2013: Laser Sensing and Imaging and Applications, 8905, p. 89052Y, International Society for Optics and Photonics, 2013.

[17] J. A. Ogilvy, Theory of wave scattering from random rough surfaces, A. Hilger, Bristol, England Philadelphia, 1991.

[18] P. Beckmann, The scattering of electromagnetic waves from rough surfaces, Artech House, Norwood, MA, 1987. 